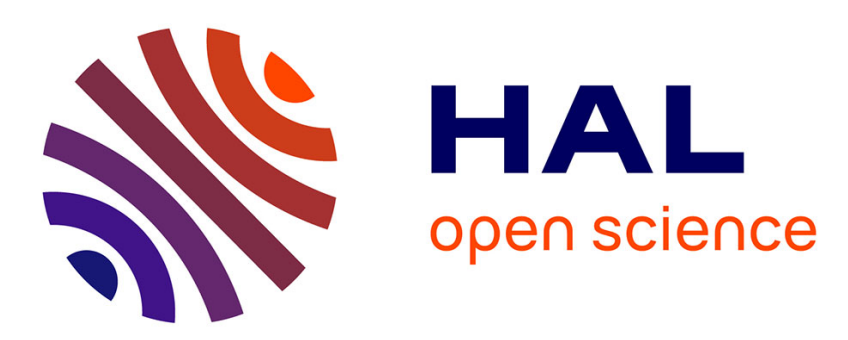

\title{
A computational strategy for poroelastic problems with a time interface between coupled physics
}

David Néron, David Dureisseix

\section{To cite this version:}

David Néron, David Dureisseix. A computational strategy for poroelastic problems with a time interface between coupled physics. International Journal for Numerical Methods in Engineering, 2008, 73 (6), pp.783-804. 10.1002/nme.2091 . hal-00322483

\section{HAL Id: hal-00322483 \\ https://hal.science/hal-00322483}

Submitted on 18 May 2015

HAL is a multi-disciplinary open access archive for the deposit and dissemination of scientific research documents, whether they are published or not. The documents may come from teaching and research institutions in France or abroad, or from public or private research centers.
L'archive ouverte pluridisciplinaire HAL, est destinée au dépôt et à la diffusion de documents scientifiques de niveau recherche, publiés ou non, émanant des établissements d'enseignement et de recherche français ou étrangers, des laboratoires publics ou privés. 


\title{
A computational strategy for poroelastic problems with a time interface between coupled physics
}

\author{
D. Néron ${ }^{1}$ and D. Dureisseix ${ }^{2}$
}

${ }^{1}$ LMT-Cachan (ENS de Cachan / CNRS UMR 8535 / Paris 6 University) 61, avenue du Président Wilson, F-94235 CACHAN CEDEX, FRANCE

2 LMGC (Montpellier 2 University / CNRS UMR 5508 CC 048, place Eugène Bataillon, F-34095 MONTPELLIER CEDEX 5, FRANCE

\begin{abstract}
This paper deals with a computational strategy suitable for the simulation of multiphysics problems and based on the Large Time INcrement (LATIN) method. One of the main issues in the design of advanced tools for the simulation of such problems is to take into account the different time and space scales which usually arise with the different physics. Here, we focus on using different time discretizations for each physic by introducing an interface with its own discretization. The proposed application concerns the simulation of a 2-physics problem: the fluid-structure interaction in porous media.

This is the post-print accepted version of the following article: D. Néron, D. Dureisseix, A computational strategy for poroelastic problems with a time interface between coupled physics, International Journal for Numerical Methods in Engineering 23(6):783-804. Wiley, 2007, DOI: 10.1002/nme.2091, which has been published in final form at

http://onlinelibrary.wiley.com/doi/10.1002/nme.2091/abstract
\end{abstract}

Keywords: multiphysics; poroelasticity; partitioning; LATIN; multiscale; finite elements; non matching grids

\section{INTRODUCTION}

\subsection{Simulation of coupled multiphysics problems}

Recently, a computational strategy based on the LArge Time INcrement method (LATIN) [1] dealing with coupled multiphysics problems has been designed as a partitioning strategy [2] and applied to poroelasticity problems before being extended to the general case of problems described in a discretized form $[3]$.

The chosen partitioning is to separate the physics in order to avoid the simultaneous treatment of the different fields, as in $[4,5,6,7,8,9,10]$ for similar or other fluid-structure interaction problems. Another way of partitioning, when the couplings occur in the body of the domain, is to split geometrically the domain, as in classical domain decomposition methods [11, 12], and to keep the local problems coupled, as in $[13,14,15,16]$.

One advantage of the first approach resides in its modularity, allowing different treatments (different softwares, time and space discretizations, integration schemes) for the different physics involved [8, 17 , $18,19,20,21]$. However, if the different softwares use their own meshing (or remeshing) ability, the issue is then to be able to deal with incompatible (or non-matching) time and space discretizations for the simulation.

The feasibility of the LATIN approach for multiphysics problems was presented in [2]. It concerned the simulation of a 2-physics problem: the saturated poroelastic transient evolution of a media in which the physics involved were the fluid phase in connected porosity and the solid phase as the skeleton of the porous media; both were homogenized $[9,22,23]$ to give rise to a strongly coupled multifield problem. For the same problem, the possibility of coupling two different time discretizations was described in [24], using a fixed point method between the two physics to ensure the verification of the coupled equations. The convergence of this fixed point method had not been proved at that time, but was assessed in various 
numerical test cases. However, such a procedure requires numerous field transfer operations between the physics, which can lead to expensive computations, especially for a more-than-2-physics problem. This is the first motivation to enhance the approach already developed by introducing the notion of interface between physics. This point is the subject of this article in which, for sake of simplicity, the notion of interface between physics is presented on a monodimensional poroelastic consolidation problem when different time discretizations are coupled. Several approaches may be designed: the use of a fixed point method between two discretizations is recalled, a convergence analysis is now given, and an alternative method based on an interface between the physics is proposed and discussed. The main advantages of the last approach are the modularity and the cost reduction, especially with both non matching time and space discretizations for which the transfer of space-time fields from one discretization to the another will be a more costly operation. The case of non matching space discretizations is currently under development.

\subsection{An interface between physics}

For sake of simplicity in this Section, the presentation is restrained to the case of a 2-physics problem without loosing its generality. In this article, we are concerned with multiphysics problems for which the unknowns associated to each physics are assumed to be defined over the whole time-space domain $[0, T] \times \Omega$ and the coupling to be defined within the constitutive relations. This implies that the coupled problem to be solved is defined over the whole time-space domain $[0, T] \times \Omega$. The point of view adopted herein is to extend the notion of geometrical interface used for domain decomposition methods in [25] (see Figure 1), to the concept of an interface between different physics (see Figure 2).

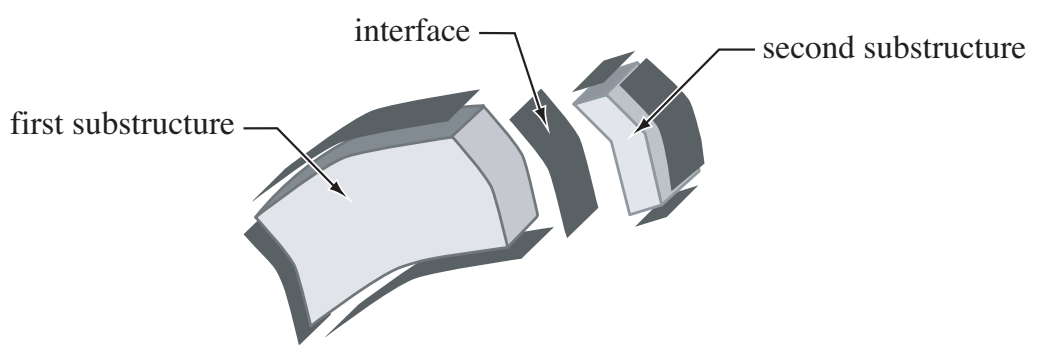

Figure 1: Geometrical interface for domain decomposition

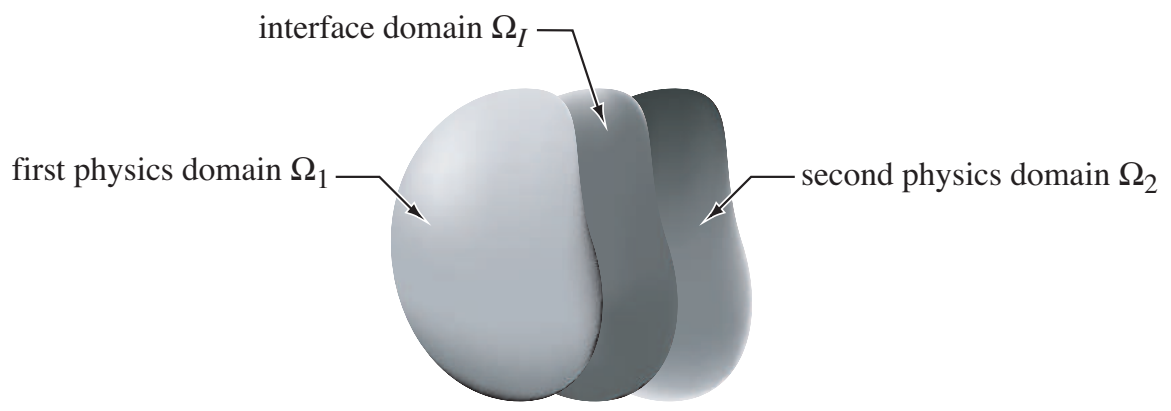

Figure 2: Interface between physics defined on the whole domain

This 'interface between physics' is defined on the time-space domain $T_{I} \times \Omega_{I}\left(T_{I}\right.$ is a possible time discretization of this interface and $\Omega_{I}$ is the corresponding space discretization). Its behavior consists in the verification of the constitutive relations which couple the physics. Time-space domain $T_{1} \times \Omega_{1}$ (respectively $T_{2} \times \Omega_{2}$ ) on which only the part $\mathbf{s}_{1}$ (respectively $\mathbf{s}_{2}$ ) of the unknowns corresponding to the first (respectively second) physics, is defined. Its behavior consists in letting these unknowns satisfy the compatibility conditions and the conservation laws.

Using such an interface allows to get modularity in the solution procedure, as well as in the modeling of the coupled phenomena. For instance, if a physical model changes, or if a third physics is added, a corresponding time-space domain can be added, while the new couplings enrich the interface behavior. With the use of the interface, the problem can be easily expressed with a partitioning formulation. 
As the following Sections will exemplify it, this allows to choose different time discretizations and representations of the unknowns for each physics and interface. This modularity is especially interesting when different simulation codes and solvers are used for the different physics.

\section{A POROELASTIC TEST CASE}

\subsection{Problem description}

The problem we are concerned in is a saturated poroelastic structure in quasi-static and isothermal transient evolution. This is a linear problem of fluid transfer in porous media. Though non linear behaviors have already been tested for multiphysics problems with the LATIN method [26], we will focus in this Section on the linear case, while dealing with different time scales for the different physics.

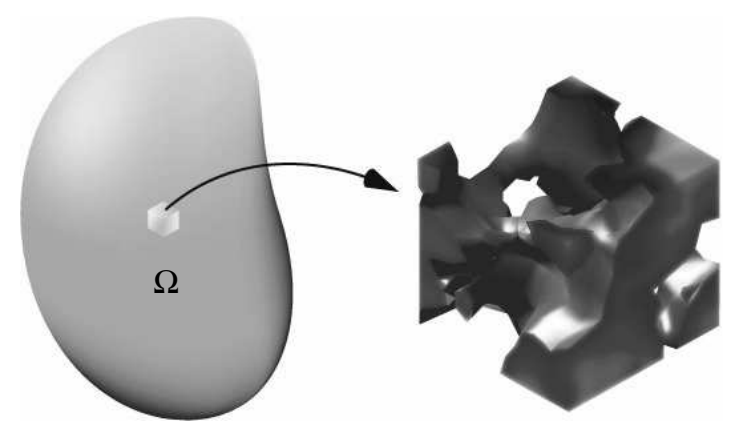

Figure 3: Internal structure of a poroelastic structure $\Omega$

Let us study the evolution over the time interval $[0, T]$ of a structure $\Omega$ made of a saturated porous material undergoing small perturbations (Figure 3). At the microscopic level, the geometry of the pores is often too complex to described the interaction between the solid and the fluid phases. Therefore, a macroscopic model is generally preferred, where the two phases are assumed to occupy the entire domain $\Omega$, forming two overlapping continuous media which can be treated with the classical methods of the mechanics of continuous porous media [9, 22, 23]. This model is build upon homogenization techniques and the macroscopic state of the poroelastic structure is described using the set of fields $\mathbf{s}=(\dot{\varepsilon}, \boldsymbol{\sigma}, p, q, \underline{Z}, \underline{W})$, defined on the whole time-space domain $[0, T] \times \Omega$, and where the quantities involved are:

- for the solid part (the ' $S$-physics'): the strain field $\varepsilon$ and the stress field $\boldsymbol{\sigma}$;

- for the fluid part (the ' $F$-physics'): the pore pressure field $p$, its gradient $\underline{Z}$, the opposite of Darcy's velocity $\underline{W}$ and the rate of fluid accumulation $q$ in each representative elementary volume.

This homogenization leads to a fluid-structure interaction problem where the coupling between the two physics occurs in the whole body $\Omega$. Nevertheless, even at the macro scale, different time and/or space lengths can be exhibited for each physics and different discretizations can be chosen accordingly.

\subsection{Coupled constitutive relations and field admissibility}

The macroscopic constitutive relations, which link the $S$-physics and the $F$-physics, are local in space and coupled. For an isotropic behavior, at each point $M \in \Omega$, and for each time step $t \in[0, T]$, one gets:

- Hooke's law, which relates the stress $\boldsymbol{\sigma}$ to the strain $\varepsilon$ and the pore pressure $p$ :

$$
\boldsymbol{\sigma}=\mathbf{D} \boldsymbol{\varepsilon}-b p \mathbf{I}
$$

- Darcy's law, which relates Darcy's velocity $-\underline{W}$ to the pore pressure gradient $\underline{Z}$ :

$$
\underline{W}=H \underline{Z}
$$


- and finally, the compressibility law, which relates the fluid accumulation rate $q$ to the pressure rate $\dot{p}$ and couples it with the rate of volume modification $\operatorname{Tr} \dot{\varepsilon}$ :

$$
q=\frac{1}{Q} \dot{p}+b \operatorname{Tr} \dot{\varepsilon}
$$

In these relations, $\mathbf{D}$ is Hooke's tensor of the drained skeleton, $b$ is Biot's coefficient, $H=\frac{K}{\mu_{w}}$ is the permeability of the porous media ( $K$ is the intrinsic macroscopic permeability, $\mu_{w}$ is the dynamic viscosity of the saturation fluid) and $Q$ is Biot's modulus.

The admissibility conditions of the different fields are global in space but decoupled between the two physics and have been detailed in [2]. They will only be recalled in the particular example which is the subject of the following Subsections.

\subsection{A monodimensional problem as test case}

In the following, we describe a monodimensional problem as an illustrative example, useful to derive some characteristics of the proposed approach, and to settle the parameters that may influence the choice of the time discretizations. The studied domain $\Omega$ is a monodimensional medium of length $\ell$ (the characteristic spatial length of the phenomenons that wish to be captured) represented Figure 4 . One end of the medium is clamped, the other one is subjected to an external traction stress $\sigma_{d}(t)$. The pore pressure is prescribed on both ends to be $p_{1}(t)$ and $p_{2}(t)$. Finally, the initial pore pressure is $p_{0}(x)$, where $x$ is the spatial coordinate.

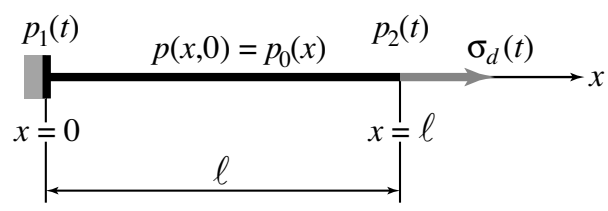

Figure 4: Monodimensional problem

All the previous fields degenerate into scalar fields and, with obvious notations, $E$ being Young modulus, the corresponding constitutive relations read:

$$
\begin{aligned}
\sigma & =E \varepsilon-b p \\
W & =H Z \\
q & =\frac{1}{Q} \dot{p}+b \dot{\varepsilon}
\end{aligned}
$$

For $S$-physics fields, the equilibrium of the stress reads $\frac{\partial \sigma}{\partial x}=0$ with $\sigma(\ell, t)=\sigma_{d}(t)$, which leads to $\sigma(x, t)=\sigma_{d}(t)$; for $F$-physics fields, the compatibility of the pressure gradient is $Z=\frac{\partial p}{\partial x}$ and the flow conservation $q=\frac{\partial W}{\partial x}$, the boundary conditions are $p(0, t)=p_{1}(t)$ and $p(\ell, t)=p_{2}(t)$; finally, the initial condition is $p(x, 0)=p_{0}(x)$.

\subsection{A reference solution}

In this subsection, we propose to seek a simple analytical solution of the previous problem, in order to be used as a reference solution for the solving strategy which is the topic of this paper and will be introduced in the following. For sake of simplicity and to make this reference solution as simple as possible, we will choose the more convenient boundary conditions.

Obviously, the statical admissibility for the stress provides $\sigma=\sigma_{d}(t)$; therefore, Hooke's law gives $\varepsilon=\frac{1}{E}\left(\sigma_{d}+b p\right)$.

Fields $q, W$ and $Z$ can be substituted using the other admissibility conditions and Darcy's law in the last constitutive relation. This leads to the linear ordinary differential equation (ODE) for the pore pressure:

$$
H \frac{d^{2} p}{d x^{2}}-\left(\frac{1}{Q}+\frac{b^{2}}{E}\right) \dot{p}=\frac{b}{E} \dot{\sigma}_{d}
$$


for which a physical solution (with separated variables) is:

$$
p(x, t)=e^{-t / \tau_{F}}\left[P_{1} \cos (\omega x)+P_{d} \sin (\omega x)\right]-\frac{\frac{b}{E}}{\frac{1}{Q}+\frac{b^{2}}{E}} \sigma_{d}+p_{d}
$$

where:

$$
\tau_{F}=\frac{1}{H}\left(\frac{1}{Q}+\frac{b^{2}}{E}\right) \frac{1}{\omega^{2}}
$$

$\tau_{F}$ is the characteristic time for the $F$-physics; $P_{1}, P_{d}, \omega$ and $p_{d}$ are constants to be determined by boundary and initial conditions, to satisfy the whole set of equations.

To get a simple solution, one may choose to set $p_{d}=\sigma_{d}(t=0)=0, P_{1}=0$, and $\sin (\omega \ell)=0$ which relates the pulsation to the characteristic length: $\omega=\frac{\pi}{\ell}$, provided that:

$$
p_{1}(t)=p_{2}(t)=-\frac{\frac{b}{E}}{\frac{1}{Q}+\frac{b^{2}}{E}} \sigma_{d}(t)
$$

and

$$
p_{0}(x)=P_{d} \sin (\omega x)
$$

A back substitution recovers all the other fields of the problem. In particular, one gets:

$$
\begin{aligned}
& p=P_{d} e^{-t / \tau_{F}} \sin (\omega x)-\frac{\frac{b}{E}}{\frac{1}{Q}+\frac{b^{2}}{E}} \sigma_{d}(t) \\
& \varepsilon=\frac{b}{E} P_{d} e^{-t / \tau_{F}} \sin (\omega x)+\frac{\frac{1}{Q}}{\frac{1}{Q}+\frac{b^{2}}{E}} \frac{\sigma_{d}(t)}{E}
\end{aligned}
$$

One can notice that

- two characteristic times are involved in the solution: $\tau_{F}$ is the characteristic time of the $F$-physics, while the characteristic time of the $S$-physics is given by the loading $\sigma_{d}(t)$;

- the amplitudes of the two contributions in each field are driven by the coupling coefficient $b$;

- the evolutions can be represented as a sum of two products of a scalar time evolution by a space field. The two space fields are $\sin (\omega x)$ and a constant unitary field.

\subsection{A simple test problem with time evolutions as unknowns}

Up to now, we wish to derive a simple problem, focussing on time evolutions, to introduce the iterative solving strategy and the coupling of different time discretizations for the different physics. Therefore, we assume that the spatial patterns of the various fields are prescribed to be those of the analytical solution, and we propose to set the unknowns as the time evolutions only. The previous fields are now searched as:

$$
\begin{aligned}
\varepsilon(x, t) & =\left[\begin{array}{ll}
\sin (\omega x) & 1
\end{array}\right]\left[e_{1}(t) e_{2}(t)\right]^{T}=\sin (\omega x) e_{1}(t)+e_{2}(t) \\
\sigma(x, t) & =\left[\begin{array}{ll}
\sin (\omega x) & 1
\end{array}\right]\left[\begin{array}{ll}
s_{1} & s_{2}
\end{array}\right]^{T} \\
p(x, t) & =\left[\begin{array}{ll}
\sin (\omega x) & 1
\end{array}\right]\left[\begin{array}{ll}
\pi_{1} & \pi_{2}
\end{array}\right]^{T} \\
q(x, t) & =\left[\begin{array}{ll}
\sin (\omega x) & 1
\end{array}\right]\left[\theta_{1} \theta_{2}\right]^{T} \\
Z(x, t) & =\left[\begin{array}{ll}
\cos (\omega x) & 1
\end{array}\right]\left[\begin{array}{ll}
z_{1} & z_{2}
\end{array}\right]^{T} \\
W(x, t) & =\left[\begin{array}{ll}
\cos (\omega x) & 1
\end{array}\right]\left[\begin{array}{ll}
w_{1} & w_{2}
\end{array}\right]^{T}
\end{aligned}
$$

As the spatial fields are given, the problem is to find $\mathbf{s}=\left(\mathbf{s}_{S}, \mathbf{s}_{F}\right)$ with $\mathbf{s}_{S}=\left\{\left(\dot{e}_{i}, s_{i}\right), i=1,2\right\}$ and $\mathbf{s}_{F}=\left\{\left(\pi_{i}, \theta_{i}, z_{i}, w_{i}\right), i=1,2\right\}$, the set of unknown time functions satisfying the constitutive relations: for $i=1,2$,

$$
\begin{array}{r}
s_{i}=E e_{i}-b p_{i} \\
w_{i}=H z_{i} \\
\theta_{i}=\frac{1}{Q} \dot{p}_{i}+b \dot{e}_{i}
\end{array}
$$


the admissibility conditions for the $S$-physics:

$$
s_{1}(t)=0, \quad s_{2}(t)=\sigma_{d}(t)
$$

the admissibility conditions for the $F$-physics:

$$
\begin{array}{cc}
\theta_{1}(t)=-\omega w_{1}(t), & \theta_{2}(t)=0 \\
z_{1}(t)=\omega \pi_{1}(t), & z_{2}(t)=0 \\
\pi_{2}(t)=p_{1}(t)=p_{2}(t)
\end{array}
$$

and the initial conditions: $\pi_{1}(0)=P_{d}, \pi_{2}(0)=0\left(\right.$ and $e_{i}(0)=-\frac{b}{E} \pi_{i}(0)$ whenever needed $)$.

\section{THE LARGE TIME INCREMENT METHOD AS A SOLVER}

The Large Time INcrement method (LATIN) is a general mechanics-based computational strategy originally designed for solving time-dependent nonlinear problems [1]. The extension of this strategy for multiphysics problems has been reported in $[2,24,26]$. Its main originality lies in a non-incremental iterative approach which operates over the entire time-space domain. This method has been successfully applied to various quasi-static and dynamic problems, post-buckling analysis and domain decomposition $[27,28,29,30,31,32,33]$. The next three Subsections briefly recall this approach. Arguments to select the search direction parameters are then settled, and the following discussion focuses on the time discretization coupling with dedicated tools to manage different discretizations within the framework of time discontinuous Galerkin approaches.

\subsection{Principles of the method}

The solution of the reference problem has to verify the following equations:

- the admissibility of the $S$-physics (13);

- the admissibility of the $F$-physics (14);

- the behavior of the interface between the $S$ and the $F$-physics (12), and the initial conditions.

To design the solver, 2 principles of the LATIN method are used:

- the first principle consists in splitting the difficulties to avoid the simultaneous treatment of two difficulties: globality of the equations to solve and coupling between the physics. A first set of equations, $\mathbf{A}_{\mathbf{d}}$, contains the global but uncoupled field admissibilities of the $S$ and the $F$-physics (13),(14). A second set, $\boldsymbol{\Gamma}$, contains the local and coupled behavior of the interface between the physics (12), together with initial conditions. Thus, the solution $\mathbf{s}_{e x}$ of the problem is the intersection of $\mathbf{A}_{\mathbf{d}}$ and $\boldsymbol{\Gamma}$;

- the second principle consists in using an iterative procedure, producing alternatively elements of $\boldsymbol{\Gamma}$ and $\mathbf{A}_{\mathbf{d}}$, up to the convergence. Iteration $n+1$ contains two stages (see Figure 5): (i) once a solution of $\mathbf{A}_{\mathbf{d}}$ is known, the local stage consists in finding a solution of $\boldsymbol{\Gamma}$, using an upward search direction $\mathbf{E}^{+}$, (ii) once a solution of $\boldsymbol{\Gamma}$ is known, the decoupled stage consists in finding a solution of $\mathbf{A}_{\mathbf{d}}$, using a downward direction $\mathbf{E}^{-}$. The particular expressions of these search directions will be precised in the following Subsections. If they are constant along iterations, and conjugate with each other, a proof of convergence of the algorithm can be built, following [1].

$$
\cdots \longrightarrow \mathbf{s}_{n} \in \mathbf{A}_{\mathbf{d}} \underbrace{\stackrel{\text { local stage }}{\longrightarrow} \hat{\mathbf{s}}_{n+1 / 2} \in \boldsymbol{\Gamma} \stackrel{\text { decoupled stage }}{\longrightarrow}}_{\text {Iteration } n+1} \mathbf{s}_{n+1} \in \mathbf{A}_{\mathbf{d}} \longrightarrow \hat{\mathbf{s}}_{n+3 / 2} \in \boldsymbol{\Gamma} \longrightarrow \cdots \longrightarrow \mathbf{s}_{e x}
$$

Figure 5: Local and decoupled stages at Iteration $n+1$

Figure 6 attempts to offer a 'geometrical' interpretation of the method, figuring the various sets of equations $\mathbf{A}_{\mathbf{d}}, \boldsymbol{\Gamma}, \mathbf{E}^{+}$and $\mathbf{E}^{-}$in the space generated by $\left(\dot{e}_{i}, \pi_{i}, z_{i}\right), i=1,2$, and $\left(s_{i}, \theta_{i}, w_{i}\right), i=1,2$. 


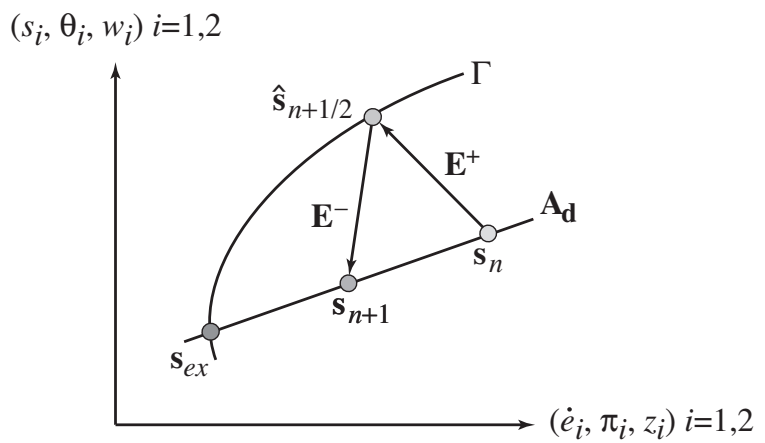

Figure 6: 'Geometrical' interpretation of the method at Iteration $n+1$

\subsection{The local stage}

Suppose $\left(\mathbf{s}_{S}, \mathbf{s}_{F}\right)$ is a known element of $\mathbf{A}_{\mathbf{d}}$, the local stage consists in finding $\hat{\mathbf{s}}$ satisfying the constitutive relations (12) at each time step. To close the problem at this stage, one has to add an upward search direction $\mathbf{E}^{+}$, whose selection has been discussed in [2]. Here, it reads:

$$
\begin{aligned}
\left(\hat{s}_{i}-s_{i}\right)+L\left(\hat{\dot{e}}_{i}-\dot{e}_{i}\right) & =0 \\
\left(\hat{\theta}_{i}-\theta_{i}\right)+r\left(\hat{\pi}_{i}-\pi_{i}\right) & =0 \\
\left(\hat{w}_{i}-w_{i}\right)+H\left(\hat{z}_{i}-z_{i}\right) & =0
\end{aligned}
$$

where $L$ and $r$ are two parameters of the method which do not influence the solution once convergence has been reached. They can be chosen of the form $L=t_{S} E$ and $r=\frac{1}{Q t_{F}} \cdot t_{S}$ and $t_{F}$ are two arbitrary durations. One can get the solution of this stage with the ODE system:

$$
\begin{aligned}
L \hat{\dot{e}}_{i}+E \hat{e}_{i}-b \hat{\pi}_{i} & =s_{i}+L \dot{e}_{i} \\
\frac{1}{Q} \dot{\hat{\pi}}_{i}+r \hat{\pi}_{i}+b \hat{\dot{e}}_{i} & =\theta_{i}+r \pi_{i}
\end{aligned}
$$

with initial conditions on $\pi_{i}$ and $e_{i}$, and:

$$
\hat{w}_{i}=H \hat{z}_{i}=\frac{1}{2}\left(w_{i}+H z_{i}\right)
$$

\subsection{The decoupled stage}

Suppose $\hat{\mathbf{s}}$ is a known element of $\boldsymbol{\Gamma}$, the decoupled stage consists in finding $\left(\mathbf{s}_{S}, \mathbf{s}_{F}\right)$ in $\mathbf{A}_{\mathbf{d}}$ which verify the decoupled admissibility conditions (13) and (14). A downward search direction $\mathbf{E}^{-}$is used, conjugate to the previous one (since both $\theta_{2}$ and $\pi_{2}$ are given by admissibility conditions, no search direction is used for them).

$$
\begin{aligned}
\left(s_{i}-\hat{s}_{i}\right)-L\left(\dot{e}_{i}-\hat{\dot{e}}_{i}\right) & =0 \\
\left(\theta_{1}-\hat{\theta}_{1}\right)-r\left(\pi_{1}-\hat{\pi}_{1}\right) & =0 \\
\left(w_{i}-\hat{w}_{i}\right)-H\left(z_{i}-\hat{z}_{i}\right) & =0
\end{aligned}
$$

Apart from the unknowns directly given by admissibility conditions, the solution at this stage is:

$$
\begin{aligned}
\dot{e}_{i} & =\hat{\dot{e}}_{i}+L^{-1}\left(s_{i}-\hat{s}_{i}\right) \quad \text { for } i=1,2 \\
\pi_{1} & =\frac{1}{r+H \omega^{2}}\left[\left(r \hat{\pi}_{1}-\hat{\theta}_{1}\right)+\omega\left(H \hat{z}_{1}-\hat{w}_{1}\right)\right] \\
w_{1} & =\frac{1}{r+H \omega^{2}}\left[H \omega\left(r \hat{\pi}_{1}-\hat{\theta}_{1}\right)-r\left(H \hat{z}_{1}-\hat{w}_{1}\right)\right]
\end{aligned}
$$

\subsection{Choice of search direction parameters}

Though the choice of $t_{F}$ and $t_{S}$ does not influence the solution at convergence, the rate of convergence may change along with their values. On the previous simple test, one can assess the convergence rate 
when considering the iteration matrix giving $\mathbf{s}_{n+1}$ once $\mathbf{s}_{n}$ is known, and minimizing its spectral radius with respect to the search direction parameters $t_{F}$ and $t_{S}$. With the previous explicit expressions of the solution after a decoupled and a local stage, one gets after algebraic manipulations:

$$
\left[\begin{array}{l}
\theta_{1}+r \pi_{1} \\
\theta_{2}+r \pi_{2}
\end{array}\right]_{n+1}=\left[\begin{array}{cc}
H \omega^{2}-r & 0 \\
H \omega^{2}+r & 0 \\
0 & 0
\end{array}\right]\left[\begin{array}{l}
\hat{\theta}_{1}-r \hat{\pi}_{1} \\
\hat{\theta}_{2}-r \hat{\pi}_{2}
\end{array}\right]+\underline{A}
$$

where $\underline{A}$ is a constant given vector (which depends in particular on the initial conditions of the problem) and $\left(\hat{\theta}_{i}-r \hat{\pi}_{i}\right)$ can themselves be expressed as a function of $\left(\theta_{i}+r \pi_{i}\right)_{n}$ and $\left(s_{i}, e_{i}\right)_{n}$. Minimizing the spectral radius of the iteration matrix encourages to select $H \omega^{2}-r=0$, i.e. $t_{F}=\frac{1}{H Q \omega^{2}}$ which is close to $\tau_{F}$.

The situation is less clear for the solid part, since there is no physical time associated to the decoupled solid behavior (no viscosity, no dynamical evolution). Therefore, one can choose for the search direction parameter $t_{S}$ either the characteristic time of the fluid in the case of strong coupling, or a characteristic time of the loading (here, $\sigma_{d}(t)$ ). The numerical study of the convergence rate with respect to the search direction parameters is not under the scope of this paper.

\subsection{Time discretizations}

Let us now consider the case where different time discretizations are selected for the different physics. This could be motivated by the usage of different simulation codes, each involving its own time adaptation strategy, or by an a priori choice based on the expected gradients in the solution by the different time evolutions, that may not appear at the same time instants for the different physics, or even when the required precision on each field is different.

A time transfer operator will obviously be needed in order to exchange information between the different time grids. Such a feature was already tested in [24], but it involved a global solve on the whole time grids in order to transfer information. Here, we wish to use an approach derived from the space transfer operators discussed in [34], that leads to element by element computations. The principle is to extract the averaged evolution $e(t)$ of a field $E(t)$, Figure 7 ; the standard average on a time slab, as well as higher order averages, are discontinuous from a time slab to the other.

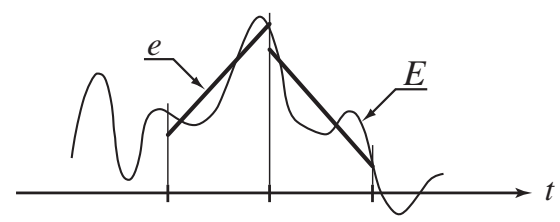

Figure 7: Generalized averaged function $e$ of a time function $E$

A time representation that allows such jumps in the solution is the time discontinuous Galerkin (TDG) approach, see $[35,36]$. Briefly, one considers a field $e(t)$ that is represented on a time interval $[0, T]$, with linear (for degree one interpolation) evolution on each time step $\left[t_{i}, t_{i+1}\right]$ (for $i=0 \ldots n, t_{0}=0$, $t_{n}=T$ ), but with possible jumps at time instants $t_{i}$. Clearly, the representation of the evolution on each time step requires two values: $e_{i}^{+}=\lim _{t \rightarrow t_{i}^{+}} e(t)$ and $e_{i+1}^{-}=\lim _{t \rightarrow t_{i+1}^{-}} e(t)$, see Figure 8 .

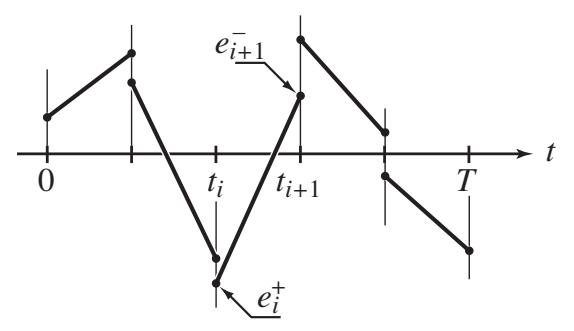

Figure 8: Degree one time discontinuous Galerkin representation 
The derivative $\dot{e}$ is not defined at these particular instants. Therefore, all of the operations involving such a quantity have to be expressed in a variational form, where the following integral is usually defined as:

$$
\int_{0}^{T} \dot{e} e^{\star} d t:=\sum_{i=0}^{n-1}\left\{\int_{\left[t_{i}, t_{i+1}\right]} \dot{e} e^{\star} d t+\left[e_{i}^{+}-e_{i}^{-}\right] e_{i}^{\star,+}\right\}
$$

for all fields $e^{\star}$ defined with the same time discontinuous approximation as for $e, e_{0}^{-}$being defined as the initial condition.

A first order ODE of the form $\dot{e}+a e=b(t)$, when using a test function $e^{\star}$ with $\left[t_{i}, t_{i+1}\right]$ as support leads to:

$$
\int_{\left[t_{i}, t_{i+1}\right]} \dot{e} e^{\star} d t+e_{i}^{+} e_{i}^{\star,+}+\int_{\left[t_{i}, t_{i+1}\right]} a e e^{\star} d t=\int_{\left[t_{i}, t_{i+1}\right]} b(t) e^{\star} d t+e_{i}^{-} e_{i}^{\star,+}
$$

which is a coupled system with $e_{i}^{+}$and $e_{i+1}^{-}$as unknowns. For second order ODE, the reader can refer to $[37,38]$ for instance.

In order to simplify the notations, in all of the following, the expressions will not be stated with this variational framework. Nevertheless, the time discontinuous Galerkin approach is used, both for the representation of the time evolution, and as a time integration scheme.

The next Subsections present the corresponding time transfer operator, and recall two algorithmic versions: with and without an additional interface discretization. Their advantages and drawbacks are also stated.

\subsection{Time transfer operator}

Consider the case of transfer between two time discretizations, denoted with $T_{1}$ and $T_{2}$, of the same time interval $[0, T]$. Using the scalar product of time functions over $[0, T]$, if we consider the transfer of $e_{1}(t)$, defined on $T_{1}$, to $e_{2}(t)$, defined on $T_{2}$, we choose to express the conservation of the scalar product with respect to a set of test functions $e_{2}^{\star}(t)$ as:

$$
\int_{T_{2}} e_{2}^{\star} e_{2} d t=\int_{T_{1}} e_{2}^{\star} e_{1} d t
$$

which can be interpreted as the conservation of generalized averages of $e_{1}$ with respect to the set of test functions. We denote with $\boldsymbol{n}_{i}(t)$ the set of the basis functions generating $T_{i}$ (in this case of discontinuous functions, $\boldsymbol{n}_{i}(t)$ is constituted by the restriction to each time step of the classical basis functions used in the continuous case). Then $e_{i}(t)=\boldsymbol{n}_{i}(t) E_{i}$ where $E_{i}$ is the column vector of the coordinates in this basis. The proposed test function basis is $\boldsymbol{n}_{2}(t)$ (see Figure 9 ), which leads to:

$$
E_{2}=\boldsymbol{p}_{21} E_{1} \quad \text { with } \quad \boldsymbol{p}_{21}=\boldsymbol{m}_{2}^{-1} \boldsymbol{m}_{21}
$$

where:

$$
\boldsymbol{m}_{2}=\int_{T_{2}} \boldsymbol{n}_{2}^{T} \boldsymbol{n}_{2} d t \quad \text { and } \quad \boldsymbol{m}_{21}=\int_{T_{1}} \boldsymbol{n}_{2}^{T} \boldsymbol{n}_{1} d t
$$

Computing the matrices $\boldsymbol{m}_{2}$ and $\boldsymbol{m}_{21}$ is not an issue for time discretizations; this can be viewed as a 1D finite element mesh of $[0, T]$ and treated with the same tools that are necessary for space field transfer in [34]. These operations allow to define the projector $\boldsymbol{p}_{21}$, such that $E_{2}=\boldsymbol{p}_{21} E_{1}$, as well as the dual projector $\boldsymbol{p}_{12}$, such that $E_{1}=\boldsymbol{p}_{12} E_{2}$, using in the same way $\boldsymbol{p}_{12}=\boldsymbol{m}_{1}^{-1} \boldsymbol{m}_{12}$. They require to solve problems with $\boldsymbol{m}_{1}$ or $\boldsymbol{m}_{2}$ as left hand sides, but these are very sparse due to the mono-dimensionality of the integration interval, and loosely coupled due to the discontinuous functions involved. In the following, for sake of simplicity, we will abusively use the same notations $\boldsymbol{p}_{21}$ and $\boldsymbol{p}_{12}$ to designate the transfer operators that operate on $e_{1}(t)$ to $e_{2}(t)$.

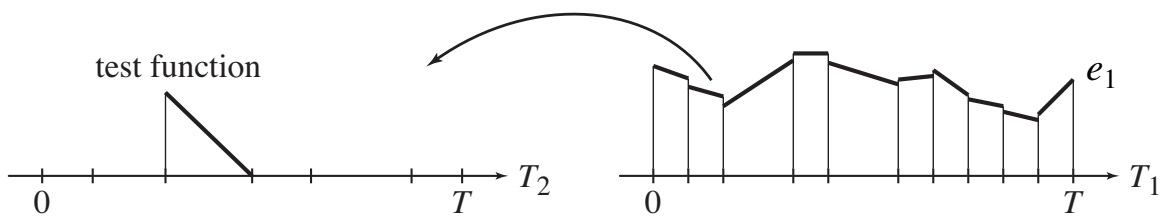

Figure 9: A proposed time discontinuous test function 
Let us mention that these projectors are closely related to mortar projectors [39, 40] and that one can prove the following properties [34]:

- conservation of scalar product: $\int_{T_{2}} e_{2} f_{2} d t=\int_{T_{1}} e_{1} f_{1} d t$ if $e_{2}=\boldsymbol{p}_{21} e_{1}$ and $f_{1}=\boldsymbol{p}_{12} f_{2}$;

- exact transfer of a field which can be represented both on $T_{1}$ and $T_{2}$.

\subsection{LATIN method with different discretizations for each physics}

This Subsection recalls the approach proposed in [24], for the 2-physics poroelastic problem, where two different time discretizations are used. A convergence proof is added, and the second approach, featuring an interface between physics will be proposed in the next Section.

The time discretization grid for the solid (respectively for the fluid) is denoted with $T_{S}$ (respectively $T_{F}$ ). The decoupled stage is related to each physics separately, therefore it deals with fields discretized on the same grid and does not require any modification.

The main adaptation lies on the local stage. It consists in finding $\mathbf{s}_{S}$ defined on the time domain $T_{S}$, and $\mathbf{s}_{F}$ defined on the other time domain $T_{F}$, satisfying the constitutive relations (12) which are now rewritten as:

$$
\hat{s}-E \hat{e}=b \boldsymbol{p}_{S F} \hat{p}
$$

and

$$
\hat{w}=H \hat{z} \quad \text { and } \quad \hat{\theta}-\frac{1}{Q} \dot{\hat{p}}=-b \frac{d}{d t}\left(\boldsymbol{p}_{F S} \hat{e}\right)
$$

where the subscript $i(i=1,2)$ has been dropped for simplicity of notations. $\boldsymbol{p}_{F S}$ (respectively $\left.\boldsymbol{p}_{S F}\right)$ is the projector from $T_{S}$ to $T_{F}$ (respectively $T_{F}$ to $T_{S}$ ). One can note that the time derivation is performed after the time projection. This allows to compute a unique projector for all the various scalar fields. Indeed, the time derivative of a field is represented in a different discrete space; if a projector has to be defined on such quantities, the previous developments should be adapted, and a specific projector derived.

Due to the projection operators, these equations are no more local in time. The search directions (15) are kept identical since they do not involve any projector (all the concerned quantities are defined on the same time domain).

The solution is given by:

$$
\hat{w}=H \hat{z}=\frac{1}{2}(w+H z)
$$

and with the coupled ODE system:

$$
\begin{aligned}
& L \hat{\dot{e}}+E \hat{e}=(s+L \dot{e})+b \boldsymbol{p}_{S F} \hat{\pi} \\
& \frac{1}{Q} \hat{\dot{\pi}}+r \hat{\pi}=(\theta+r \pi)-b \frac{d}{d t}\left(\boldsymbol{p}_{F S} \hat{e}\right)
\end{aligned}
$$

Solving directly the previous system is not so easy since the projections couple the unknowns from different time grids. A multi-rate time integration algorithm on the two grids similar as the one used in [41], for instance, could be designed, but a more trivial solution procedure is to consider a fixed point method between the two equations, each being solved on a particular time grid, provided that the convergence is ensured.

The system to solve at each step of the fixed point method is:

$$
\begin{aligned}
E\left(t_{S} \hat{\dot{e}}+\hat{e}\right) & =c+b \hat{\pi}^{p} \\
\frac{1}{Q}\left(\hat{\dot{\pi}}+\frac{1}{t_{F}} \hat{\pi}\right) & =d-b \hat{\dot{e}}^{p}
\end{aligned}
$$

where $c$ and $d$ are constant quantities during the fixed point iterations and superscript $p$ denotes the prediction arising from the previous fixed point iteration. The corresponding algorithm is described in Table 1 in which the stopping criterion of the loop is based on the monitoring of the stationarity of the fixed point method.

A proof of convergence of this fixed point method is given in the following, if the space meshes are fine enough, such that the projectors look like identity operators (almost continuous case). This proof is 


\begin{tabular}{ll}
\hline Initialization & $\hat{\pi} \leftarrow \pi$ \\
Loop on the fixed point & \\
$\quad$ Prediction & $\hat{\pi}^{p}=\boldsymbol{p}_{S F} \hat{\pi}$ \\
$\quad$ Solve on $T_{S}$ & $\hat{e} \leftarrow E\left(t_{S} \hat{\dot{e}}+\hat{e}\right)=c+b \hat{\pi}^{p}$ \\
$\quad$ Prediction & $\hat{e}^{p}=\frac{d}{d t}\left(\boldsymbol{p}_{F S} \hat{e}\right)$ \\
$\quad$ Solve on $T_{F}$ & $\hat{\pi} \leftarrow \frac{1}{Q}\left(\hat{\tilde{\pi}}+\frac{1}{t_{F}} \hat{\pi}\right)=d-b \hat{e}^{p}$ \\
$\quad$ Stopping criterion & \\
End of the loop & \\
\hline
\end{tabular}

Table 1: The fixed point method to solve the ODE system

therefore also valid for the approach used in [24], for which no proof was given at that time. Using the $\theta$-method as time integration scheme, the iteration matrix is:

$$
\left[\begin{array}{cc}
0 & b \\
-\frac{b Q}{1+\theta h / t_{F}} & 0
\end{array}\right]
$$

where $h$ is the time step length. The convergence criterion (spectral radius less than unity) leads to:

$$
b^{2}<\frac{E}{Q}\left(1+\frac{t_{S}}{\theta h}\right)\left(1+\frac{\theta h}{t_{F}}\right)
$$

In a majority of situations, $\frac{E}{Q}$ is approximately equal to 1 (it will be 1.07 in the forthcoming example of Table 2 ). Since $b \leqslant 1$, see [23], this inequality is satisfied for any values of the numerical parameters $\theta, h, t_{S}$ and $t_{F}$.

\subsection{LATIN method with a dedicated interface discretization}

The previous approach leads to an algorithm whose sketch is depicted on Figure 10, upper. The same Figure contains the corresponding sketch for a strategy using a possible third discretization for an interface (denoted by $I$ ) between the physics. The first advantage is that no fixed point iteration is required, and that the local stage is identical as for the case with a unique discretization. The integration of the small ODE required by this stage is performed only once, and the projections are performed only twice in each direction. This advantage is amplified if one considers dealing with space and time different discretizations, where the projection phase would be more costly, or when adding a new physics, as illustrated on Figure 11 with the notation $T$, where the fixed point method will be much more cumbersome.

Using a time discretization $T_{I}$ of the interface between several physics allows to deal with a different time discretization for each of them. For instance, for the previous 2-physics problem, let $T_{S}$ be the time discretization for the solid quantities, and $T_{F}$ the one of the fluid quantities. Time transfer operators are required when exchanging informations between each physics and the interface, i.e. within the search direction. We therefore propose to change the search direction of the decoupled stage (18) into:

$$
\begin{aligned}
s_{S}-L \dot{e}_{S} & =\boldsymbol{p}_{S I} \hat{s}_{I}-L \frac{d}{d t}\left(\boldsymbol{p}_{S I} \hat{e}_{I}\right) \\
\theta_{F}-r \pi_{F} & =\boldsymbol{p}_{F I}\left(\hat{\theta}_{I}-r \hat{\pi}_{I}\right) \\
w_{F}-H z_{F} & =\boldsymbol{p}_{F I}\left(\hat{w}_{I}-H \hat{z}_{I}\right)
\end{aligned}
$$

where the subscript $i(i=1,2)$ has been dropped for simplicity of notation, and where the subscripts $F$, $S$ or $I$ are used to recall the time discretization underlying a particular quantity.

The search direction of the local stage (15) is changed into:

$$
\begin{aligned}
\hat{s}_{I}+L \hat{\dot{e}}_{I} & =\boldsymbol{p}_{I S} s_{S}+L \frac{d}{d t}\left(\boldsymbol{p}_{I S} e_{S}\right) \\
\hat{\theta}_{I}+r \hat{\pi}_{I} & =\boldsymbol{p}_{I F}\left(\theta_{F}+r \pi_{F}\right) \\
\hat{w}_{I}+H \hat{z}_{I} & =\boldsymbol{p}_{I F}\left(w_{F}+H z_{F}\right)
\end{aligned}
$$




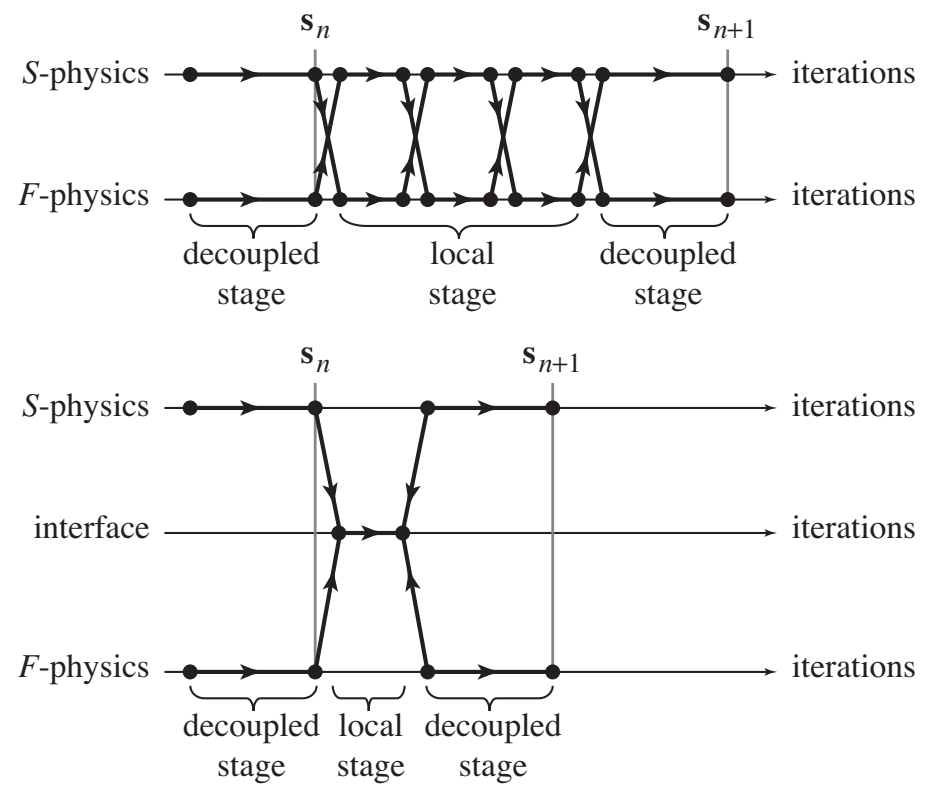

Figure 10: The algorithms for the two strategies

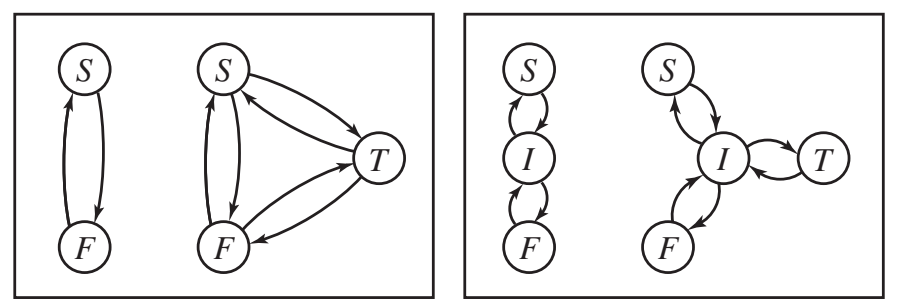

Figure 11: Modifications of transfer operations when adding a new physics 


\section{NUMERICAL RESULTS AND CHOICE OF TIME DIS- CRETIZATIONS}

\subsection{Errors to control the solution}

Once the algorithm converges, and in order to assess the quality of the solution provided, a fourth time discretization $T_{r}$ is used, much more refined than the previous one. The reference solution (denoted with a subscript $r$ ) obtained previously is computed on this last grid, and the admissible solution provided by the LATIN approach is transferred onto the same grid in order to compute the errors established in [2]. In particular, the total error is:

$$
\eta=\sqrt{\frac{\left\|\boldsymbol{p}_{r S} \varepsilon_{S}-\varepsilon_{r}\right\|_{E}^{2}+\left\|\boldsymbol{p}_{r F} p_{F}-p_{r}\right\|_{Q^{-1}}^{2}+\left\|\boldsymbol{p}_{r F} Z_{F}-Z_{r}\right\|_{H}^{2}}{\left\|\varepsilon_{r}\right\|_{E}^{2}+\left\|p_{r}\right\|_{Q^{-1}}^{2}+\left\|Z_{r}\right\|_{H}^{2}}}
$$

where, for the $S$-physics:

$$
\|\varepsilon\|_{E}^{2}=\int_{T_{r}}\left(1-\frac{t}{T}\right) \int_{0}^{\ell} \dot{\varepsilon} E \varepsilon d x d t
$$

and, for the $F$-physics:

$$
\|p\|_{Q^{-1}}^{2}=\int_{T_{r}}\left(1-\frac{t}{T}\right) \int_{0}^{\ell} \dot{p} \frac{1}{Q} p d x d t
$$

and

$$
\|Z\|_{H}^{2}=\int_{T_{r}}\left(1-\frac{t}{T}\right) \int_{0}^{\ell} Z H Z d x d t
$$

Moreover, the contribution ratio to the error of the $F$-physics and $S$-physics can be computed as:

$$
\zeta=\frac{\left\|\boldsymbol{p}_{r F} p_{F}-p_{r}\right\|_{Q^{-1}}^{2}+\left\|\boldsymbol{p}_{r F} Z_{F}-Z_{r}\right\|_{H}^{2}}{\left\|\boldsymbol{p}_{r S} \varepsilon_{S}-\varepsilon_{r}\right\|_{E}^{2}}
$$

In the numerical results obtained for the proposed test case, constant time steps are used. Therefore, there are three numerical parameters for the discretizations: $n_{S}, n_{F}$ and $n_{I}$, the number of time steps for each discretization (the reference number of time steps, $n_{r}$, is chosen much larger than the previous ones).

The loading is chosen as $\sigma_{d}(t)=P_{0} \sin \left(\omega_{S} t\right)$ for which we define the characteristic time as $\tau_{S}=\frac{\pi}{2 \omega_{S}}$.

The physical parameters of the problem are recalled in Table 2, for two cases corresponding to different ratios of characteristic times; the permeability $H$ is modified to test different $F$-physics characteristic times $\tau_{F}$, and the loading pulsation $\omega_{S}$ is modified to test different $S$-physics characteristic times $\tau_{S}$.

To avoid bad conditioning of the problem to solve, a new unit system is used, in order to have a unitary order of magnitude for $E, Q$ and $H$ : lengths are in meters $(\mathrm{L}=\mathrm{m})$, masses in $10^{10} \mathrm{~kg}\left(\mathrm{M}=10^{10}\right.$ $\mathrm{kg})$, durations in seconds $(\mathrm{S}=\mathrm{s})$.

\begin{tabular}{lll}
\hline Young modulus & $E=14.4 \mathrm{GPa}=1.44 \mathrm{M} \mathrm{L}^{-1} \mathrm{~S}^{-2}$ & \\
Biot coefficient & $b=0.4$ & \\
Biot modulus & $Q=13.5 \mathrm{GPa}=1.35 \mathrm{M} \mathrm{L}^{-1} \mathrm{~S}^{-2}$ & \\
Permeability & $H=210^{-10} \mathrm{~m}^{3} \mathrm{~s} \mathrm{~kg}^{-1}=2 \mathrm{~L}^{3} \mathrm{~S} \mathrm{M}^{-1}$ & for case 1 \\
& $H=610^{-9} \mathrm{~m}^{3} \mathrm{~s} \mathrm{~kg}^{-1}=0.6 \mathrm{~L}^{3} \mathrm{~S} \mathrm{M}^{-1}$ & for case 2 \\
Characteristic length & $\ell=1 \mathrm{~m}$ & \\
Studied time interval & $T=17310^{-3} \mathrm{~s}$ & \\
Loading levels & $P_{0}=P_{d}=20 \mathrm{GPa}=2 \mathrm{~L}^{3} \mathrm{~S} \mathrm{M}^{-1}$ & \\
$S$-physics characteristic time & $\tau_{S}=16710^{-3} \mathrm{~s}$ & for case 1 \\
& $\tau_{S}=5010^{-3} \mathrm{~s}$ & for case 2 \\
$F$-physics characteristic time & $\tau_{F}=4310^{-3} \mathrm{~s}$ & for case 1 \\
& $\tau_{F}=14410^{-3} \mathrm{~s}$ & \\
\hline
\end{tabular}

Table 2: Problem parameters 


\begin{tabular}{cccccccc}
\hline$n$ & 3 & 6 & 10 & 14 & 25 & 30 & 50 \\
$\Delta t / \mathrm{s}$ & $57.710^{-3}$ & $28.810^{-3}$ & $17.310^{-3}$ & $12.310^{-3}$ & $6.9210^{-3}$ & $5.7710^{-3}$ & $3.5610^{-3}$ \\
\hline
\end{tabular}

Table 3: Time steps values

\subsection{Optimal choice of discretizations}

All of the numbers $n$ of time steps are chosen in the set of Table 3 . Since $T=17310^{-3} \mathrm{~s}$, the same Table collects the corresponding time steps in order to be compared to the characteristic times. For the reference solution, $n_{r}=400$ and $\Delta t_{r}=0.4310^{-3} \mathrm{~s}$ ).

Two quantities are of interest: the total error $\eta$ and the contribution ratio $\zeta$. Both can be defined as a field, function of the three parameters $\left(n_{F}, n_{S}, n_{I}\right)$, representable as a 'surface' response (here, a 'volume' response) whose values are computed at each node of a $3 \mathrm{D}$ mesh in the $\left(n_{F}, n_{S}, n_{I}\right)$ space.

We propose to select the fluid and solid discretizations in order to have the same contribution to the final error. Such a criterion is represented by the iso-surface $\zeta=1$. On this surface, the error $\eta$ can be represented as a colored or a grey level map, see Figure 12, left, for the first test case, and Figure 13, left, for the second test case. Due to the interpolation between computed nodes, the surfaces are not very smooth, but a tendency can be spotted: as expected, the discretization of a physics should be refined according to the fact that the corresponding characteristic time is small. A quadratic fitting can smooth this surface and the result is depicted Figure 12, right, for the first test case, and Figure 13, right, for the second test case.

In order to get some optimal discretization rules of thumb, this last surface can be intersected by planes at constant $n_{I}$ values, see Figure 12, right. On each resulting line, the optimal point corresponds to the minimum error $\eta$. At each optimal point, Table 4 recalls the numerical characteristics of the first and second case problem (decimals in the numbers of time steps are due to the previous smoothing), and Figures 14 and 15 plot the evolutions with respect to the error $\eta$.

Clearly, the case of identical grids could not correspond to an identical error contribution for the solid and the fluid. Considering the identical grid case, for a given total error level, one has to decrease the number of time steps for one physics, and increase the number of time steps for the other to get an iso-contribution. Obviously the de-refinement of only one physics (even more of both physics) would not allow to preserve the total error value.
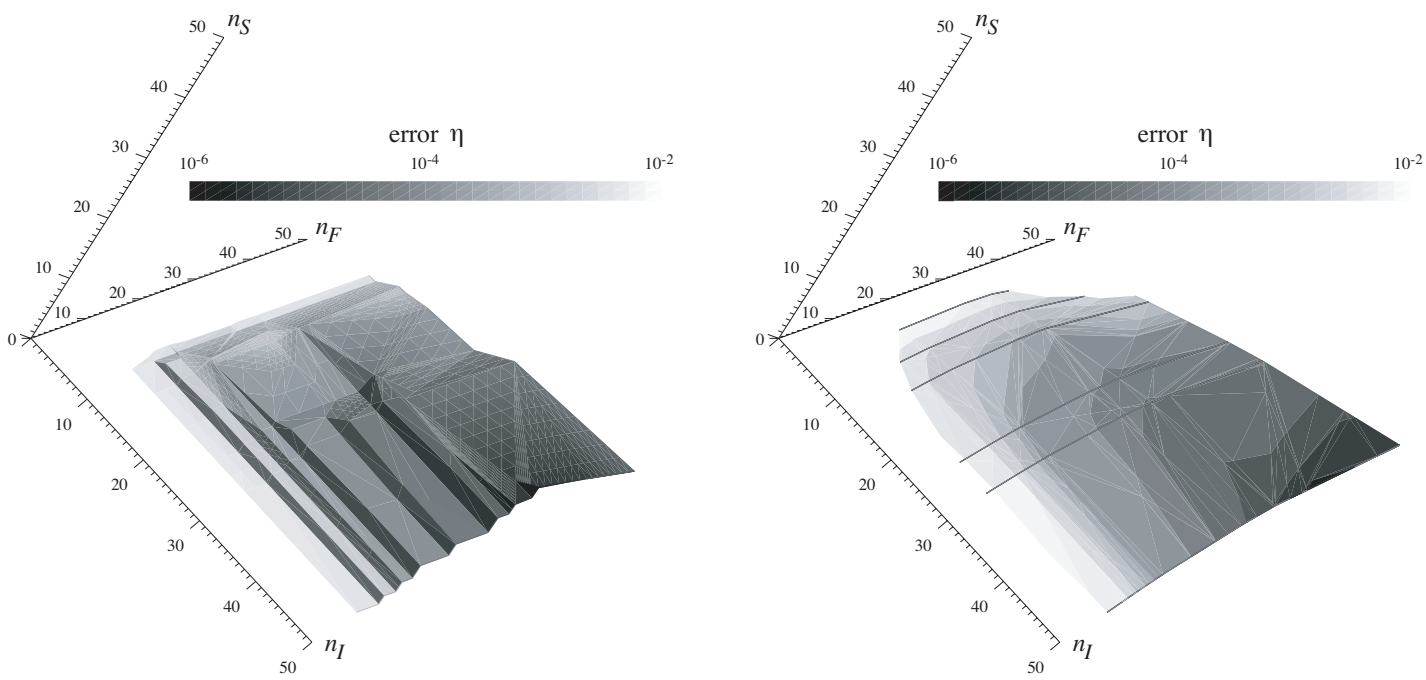

Figure 12: The iso-error contribution surfaces for the first test case (without and with a quadratic fitting)

Roughly, the ratio between the time steps is between 3 and 5 for a ratio 4 between the characteristic times for the first test case, while the ratio between the time steps is between 2 and 3 for a ratio 2.8 between the characteristic times for the second test case. Therefore, as a first approach, one can select the time step ratio as the inverse of the characteristic time ratio. The optimal interface discretization can be chosen as the average of those of the two physics. 

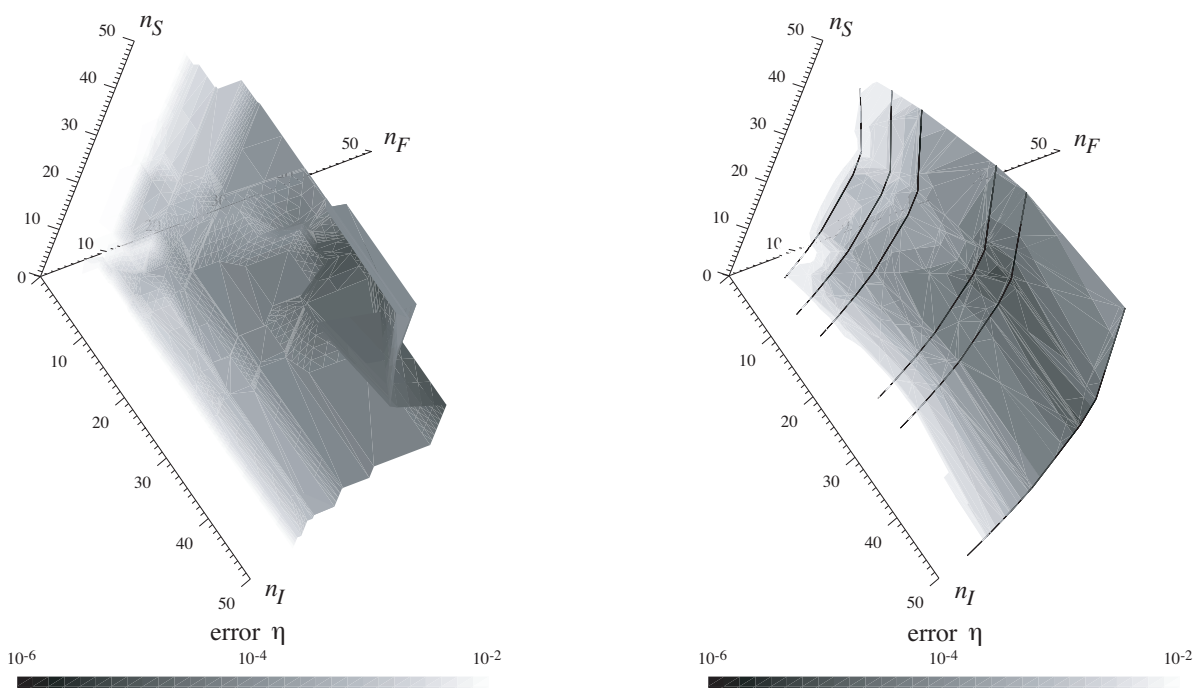

Figure 13: The iso-error contribution surfaces for the second test case (without and with a quadratic fitting)

\begin{tabular}{|c|c|c|c|c|c|}
\hline$\eta$ & $n_{I}$ & $n_{S}$ & $n_{F}$ & $n_{F} / n_{S}$ & $n_{S} / n_{F}$ \\
\hline $1.210^{-3}$ & 6 & 3.0 & 14.0 & 4.6 & \\
\hline $4.210^{-4}$ & 10 & 4.8 & 25.0 & 5.2 & \\
\hline $8.010^{-5}$ & 14 & 6.0 & 30.0 & 5.0 & \\
\hline $3.210^{-5}$ & 25 & 8.7 & 36.5 & 4.2 & \\
\hline $2.010^{-5}$ & 30 & 10.0 & 30.2 & 3.0 & \\
\hline $2.910^{-6}$ & 50 & 14.0 & 35.1 & 2.5 & \\
\hline $7.010^{-4}$ & 6 & 25.0 & 7.8 & & 3.2 \\
\hline $2.610^{-4}$ & 10 & 14.0 & 7.7 & & 1.8 \\
\hline $1.410^{-4}$ & 14 & 15.9 & 9.3 & & 1.7 \\
\hline $1.310^{-5}$ & 25 & 26.2 & 14.0 & & 1.9 \\
\hline $9.310^{-6}$ & 30 & 30.0 & 15.1 & & 2.0 \\
\hline
\end{tabular}

Table 4: Optimal discretizations for the first test case (upper part), and the second one (lower part)
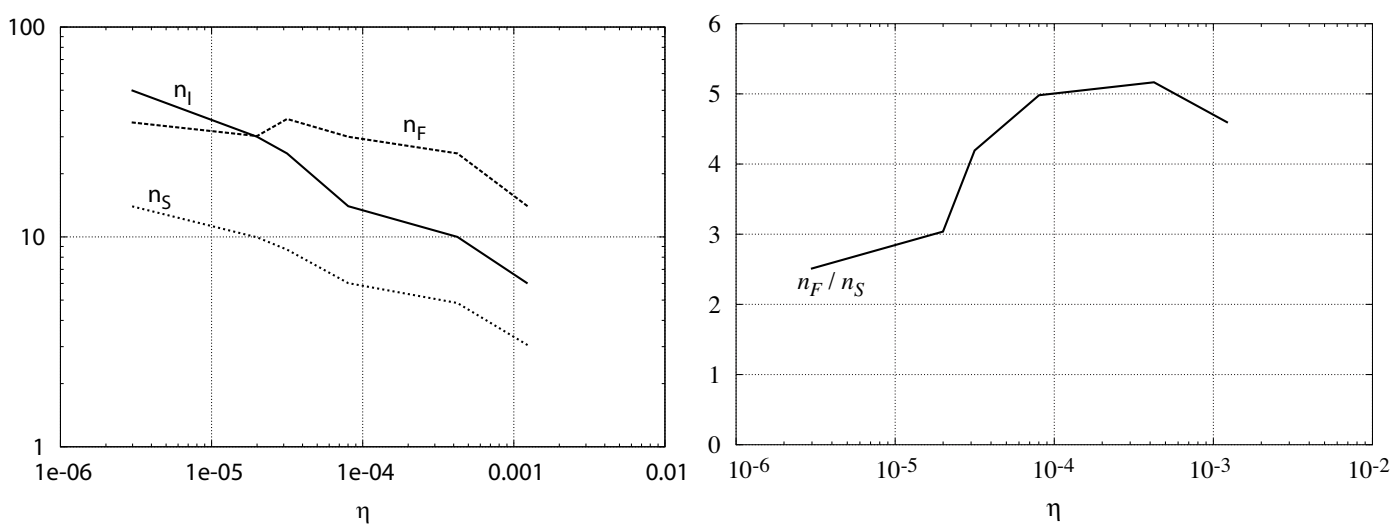

Figure 14: Optimal discretizations versus error $\eta$ for the first test case $\left(\tau_{S} / \tau_{F}=3.9\right)$ 

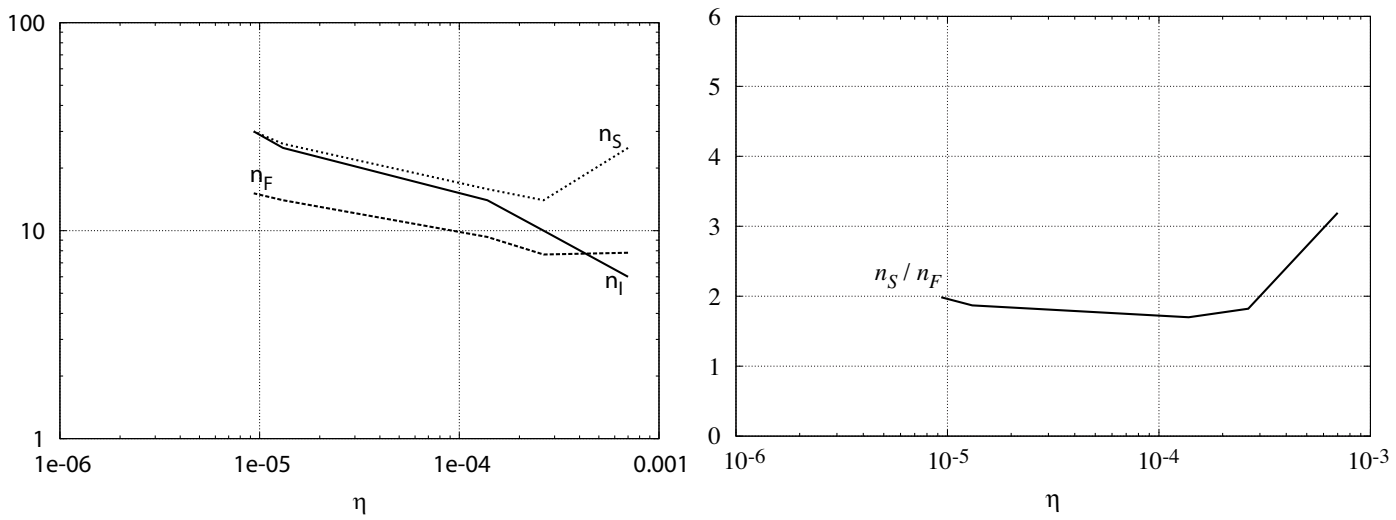

Figure 15: Optimal discretizations versus error $\eta$ for the second test case $\left(\tau_{F} / \tau_{S}=2.8\right)$

Figures 16 and 17 show the solutions obtained for the reference computation, and for the coarsest optimal discretization, for the two proposed test cases.
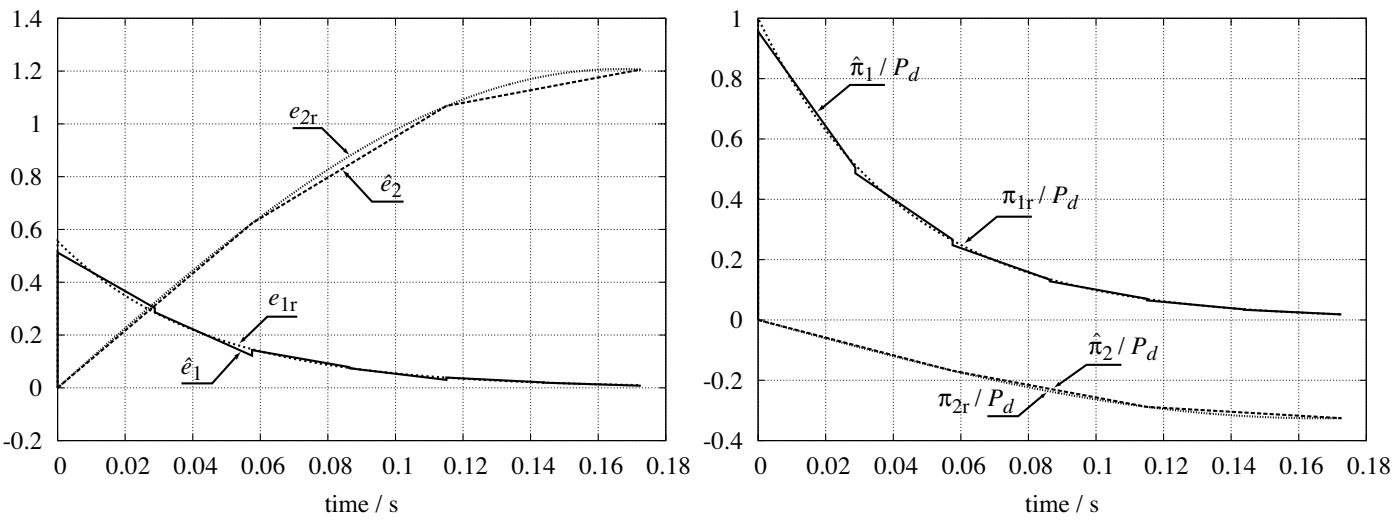

Figure 16: Solutions for the first test case; left: strain $\hat{e}_{1}$ and $\hat{e}_{2}$; right: pore pressure $\hat{\pi}_{1}$ and $\hat{\pi}_{2}$
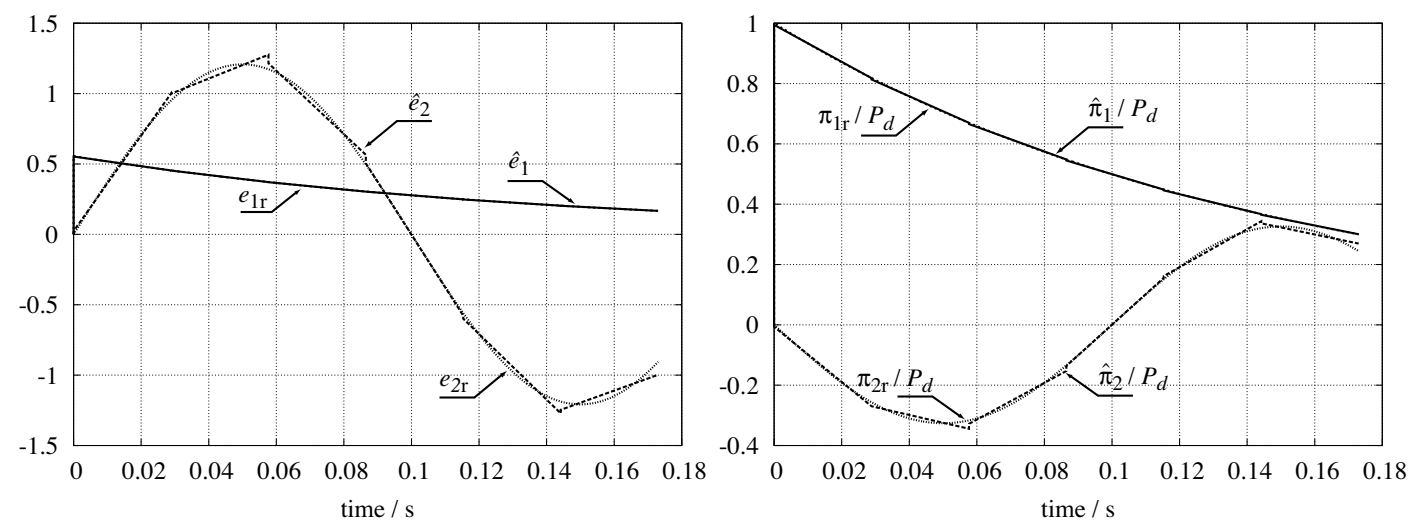

Figure 17: Solutions for the second test case; left: strain $\hat{e}_{1}$ and $\hat{e}_{2}$; right: pore pressure $\hat{\pi}_{1}$ and $\hat{\pi}_{2}$

To go a step further, some adaptive discretization procedures, as well as error estimations would be required; such topics are beyond those of this article. 


\section{CONCLUSIONS}

In this article, a computational strategy is used to solve a coupled poroelastic problem in a partitioned way. The partitioning is performed between the two involved physics, which allows to use different discretizations for the fields of each physics. We focus herein on the case of different time discretizations and we recall the possibility to use a fixed point method between the two physics to ensure the verification of the couplings (a proof of the convergence of this method is now given). Since such a procedure requires numerous field transfer operations, we introduce a specific time interface between the physics, which is the core of this article.

The influence of the numerical parameters of this approach (i.e. the various time step lengths) has been studied on a monodimensional consolidation problem, and optimal choices have been investigated. The feasibility of the transfer operators between the different discretization spaces, associated to a time discontinuous Galerkin scheme, has been exemplified.

The feasibility of the proposed approach for both time and space different discretizations, and for a more-than-2-physics coupled problem (such as a nonlinear thermo-poro-elastic transient evolution) is still to be exemplified, and is currently under development.

\section{References}

[1] P. Ladevèze. Nonlinear computational structural mechanics - New approaches and non-incremental methods of calculation. Springer Verlag, 1999.

[2] D. Dureisseix, P. Ladevèze, and B. A. Schrefler. A computational strategy for multiphysics problems - application to poroelasticity. International Journal for Numerical Methods in Engineering, 56(10):1489-1510, 2003.

[3] P. Ladevèze, D. Néron, and B. A. Schrefler. A computational strategy suitable for multiphysics problems. In Proceedings of the International Conference on Computational Methods for Coupled Problems in Science and Engineering, Santorini Island, Greece, 2005.

[4] C. A. Felippa and K. C. Park. Staggered transient analysis procedures for coupled mechanical systems: formulation. Computer Methods in Applied Mechanics and Engineering, 24:61-111, 1980.

[5] C. A. Felippa and T. L. Geers. Partitioned analysis for coupled mechanical systems. Engineering Computation, 5:123-133, 1988.

[6] R. W. Lewis, B. A. Schrefler, and L. Simoni. Coupling versus uncoupling in soil consolidation. International Journal for Numerical and Analytical Methods in Geomechanics, 15:533-548, 1991.

[7] J.-P. Morand and R. Ohayon. Fluid-structure interaction: applied numerical methods. John Wiley \& Sons, 1995.

[8] S. Piperno, C. Farhat, and B. Larrouturou. Partitioned procedures for the transient solution of coupled aeroelastic problems. Part I: model problem, theory and two-dimensional application. Computer Methods in Applied Mechanics and Engineering, 124:79-112, 1995.

[9] R. W. Lewis and B. A. Schrefler. The finite element method in the static and dynamic deformation and consolidation of porous media. John Wiley \& Sons, 2nd edition, 1998.

[10] C. Farhat and M. Lesoinne. Two efficient staggered algorithms for the serial and parallel solution of three-dimensional nonlinear transient aeroelastic problems. Computer Methods in Applied Mechanics and Engineering, 182:499-515, 2000.

[11] P. Le Tallec. Domain decomposition methods in computational mechanics. In Computational Mechanics Advances, volume 1. North-Holland, 1994.

[12] Ch. Farhat and F.-X. Roux. Implicit parallel processing in structural mechanics. In J. Tinsley Oden, editor, Computational Mechanics Advances, volume 2. North-Holland, June 1994.

[13] P. Le Tallec and A. Patra. Non-overlapping domain decomposition methods for adaptive hp approximation of the Stokes problem with discontinuous pressure fields. Computer Methods in Applied Mechanics and Engineering, 145:361-379, 1997. 
[14] C. Calgaro and J. Laminie. On the domain decomposition method for the generalized Stokes problem with continuous pressure. Numerical Methods for Partial Differential Equations, 16:84-106, 2000.

[15] P. Gosselet, Ch. Rey, P. Dasset, and F. Léné. A domain decomposition method for quasiincompressible formulations with discontinuous pressure field. Revue Européenne des Eléments Finis, 11:363-377, 2002.

[16] B. Vereecke, H. Bavestrello, and D. Dureisseix. An extension of the FETI domain decomposition method for incompressible and nearly incompressible problems. Computer Methods in Applied Mechanics and Engineering, 192:3409-3430, 2003.

[17] N. Maman and C. Farhat. Matching fluid and structure meshes for aeroelastic computations: a parallel approach. Computers and Structures, 54(4):779-785, 1995.

[18] C. Farhat, M. Lesoinne, and P. LeTallec. Load and motion transfer algorithms for fluid/structure interaction problems with non-matching discrete interfaces: Momentum and energy conservation, optimal discretization and application to aeroelasticity. Computer Methods in Applied Mechanics and Engineering, 157:95-114, 1998.

[19] A. Beckert. Coupling fluid (CFD) and structural (FE) models using finite interpolation elements. Aerospace Science and Technology, 47:13-22, 2000.

[20] C. A. Felippa, K. C. Park, and C. Farhat. Partitioned analysis of coupled mechanical systems. Computer Methods in Applied Mechanics and Engineering, 190:3247-3270, 2001.

[21] H. G. Matthies and J. Steindorf. Partitioned strong coupling algorithms for fluid-structure interaction. Computers and Structures, 81:805-812, 2003.

[22] L. Dormieux, A. Molinari, and D. Kondo. Micromechanical approach to the behavior of poroelastic materials. Journal of the Mechanics and Physics of Solids, 50:2203-2231, 2002.

[23] O. Coussy. Poromechanics. John Wiley \& Sons, 2004.

[24] D. Dureisseix, P. Ladevèze, D. Néron, and B. A. Schrefler. A multi-time-scale strategy for multiphysics problems: application to poroelasticity. International Journal for Multiscale Computational Engineering, 1(4):387-400, 2003.

[25] P. Ladevèze and D. Dureisseix. A micro/macro approach for parallel computing of heterogeneous structures. International Journal for Computational Civil and Structural Engineering, 1:18-28, 2000.

[26] D. Néron, P. Ladevèze, D. Dureisseix, and B. A. Schrefler. Accounting for nonlinear aspects in multiphysics problems: application to poroelasticity. Lecture Notes in Computer Science, 3039(IV):612$620,2004$.

[27] P.-A. Boucard, P. Ladevèze, and H. Lemoussu. A modular approach to 3-D impact computation with frictional contact. Computer and Structures, 78(1-3):45-52, 2000.

[28] H. Lemoussu, P.-A. Boucard, and P. Ladevèze. A 3-D shock computational strategy for real assembly and shock attenuator. Advances in Engineering Software, 33(7-10):517-526, 2002.

[29] P. Ladevèze, O. Loiseau, and D. Dureisseix. A micro-macro and parallel computational strategy for highly heterogeneous structures. International Journal for Numerical Methods in Engineering, 52(1-2):121-138, 2001.

[30] P. Ladevèze, A. Nouy, and O. Loiseau. A multiscale computational approach for contact problems. Computer Methods in Applied Mechanics and Engineering, 191:4869-4891, 2002.

[31] P. Ladevèze and A. Nouy. On a multiscale computational strategy with time and space homogenization for structural mechanics. Computer Methods in Applied Mechanics and Engineering, 192:3061$3087,2003$.

[32] A. Nouy and P. Ladevèze. Multiscale computational strategy with time and space homogenization: a radial-type approximation technique for solving microproblems. International Journal for Multiscale Computational Engineering, 2(4):557-574, 2004. 
[33] P. Ladevèze, D. Néron, and P. Gosselet. On a mixed and multiscale domain decomposition method. Computer Methods in Applied Mechanics and Engineering, 196:1525-1540, 2007.

[34] D. Dureisseix and H. Bavestrello. Information transfer between incompatible finite element meshes: application to coupled thermo-viscoelasticity. Computer Methods in Applied Mechanics and Engineering, 195(44-47):6523-6541, 2006.

[35] K. Eriksson, C. Johnson, and V. Thomée. Time discretization of parabolic problems by the discontinuous galerkin formulation. RAIRO Modélisation Mathématique et Analyse Numérique, 19:611-643, 1985.

[36] M. Borri and C. Bottaso. A general framework for interpreting time finite element formulations. Computational Mechanics, (13):133-142, 1993.

[37] A. Bonelli and O. S. Bursi. Iterative solutions for implicit time discontinuous Galerkin methods applied to non-linear elastodynamics. Computational Mechanics, (30):487-498, 2003.

[38] M. Mancuso and F. Ubertini. An efficient time discontinuous galerkin procedure for non-linear structural dynamics. Computer Methods in Applied Mechanics and Engineering, 195:6391-6406, 2006.

[39] C. Bernardi, Y. Maday, and A. T. Patera. A new nonconforming approach to domain decomposition: the mortar element method. In Non linear partial differential equations and their applications. Collège de France seminar, Pitman: London, 1990.

[40] C. Bernardi, Y. Maday, and A. T. Patera. A new nonconforming approach to domain decomposition: the mortar element method. In H. Brezis and J.-L. Lions, editors, Nonlinear partial differential equations and their applications, pages 13-51. Collège de France seminar, Pitman, Paris, 1994.

[41] Ch. Rey and V. Chiaruttini. An efficient multiscale parallel-in-time strategy for nonlinear poroplastic problems. In Proceedings of the 8th ESAFORM Conference on Material Forming, pages 137-140, 2005. 